\title{
Brane structure in bio-radiation
}

\author{
Bi Qiao", Song Kongzhi \\ ${ }^{1}$ Physics Dept. Wuhan University of Technology, Wuhan, China \\ ${ }^{2}$ Institute of Space Medico-Engineering, Beijing, China
}

Email address:

biqiao@gmail.com (Bi Qiao)

\section{To cite this article:}

Bi Qiao, Song Kongzhi. Brane Structure in Bio-Radiation. American Journal of Modern Physics. Vol. 3, No. 3, 2014, pp. $129-132$. doi: 10.11648/j.ajmp.20140303.12

\begin{abstract}
In this work we present a sort of structure of the brane in brane for describing the bio-radiations. The work is based on the extension of the superstring theory and can provide possible frame of biological brane to study intrinsic structure of the bio-radiations. This reveals the interaction of bio-radiation is not only electromagnetic but the week and electromagnetic, and the dimensions may be higher than four. As extension of the solitons, the brane or the brane in brane is proposed to play an important role in the bio-radiations, which can be used to explain many complicated phenomena from the somatic sciences.
\end{abstract}

Keywords: Brane, Superstring, Bio-radiation, Soliton

\section{Introduction}

Solitons in physics are solutions of equation of motion of the field theory with their energy localized. There are several works studied that the solitons are the fundamental unit of biological radiation of life, such as Davydov solitons presented by the Pan Xiaofeng [1], and composed ordered field, as our previously publication considered [2]. The general soliton has been also introduced by studying the sort of problem in bio-radiation systems [3-5].

However, the most important characteristics in the biological radiation of life research is so called "qi" from Chinese medicine and somatic science aspect, although its concept seems to imply meaning of bio-energy and information but it is never clarified to science what is exact meaning of qi. So, in this work we just explore that a possible brane structure of the bio-radiation which imply certain equivalence between brane and qi, since without this concept, many somatic phenomena are hard to understand. This is because for the specific function of human studying in the somatic science, the various of bio-radiations found play as a kind of intelligence, and contained microparticles are controlled by the mind; the relevant ray not only have kinetic energy but also information, so that the relative particles can be well according to the brain to release the energy and carry a lot of actuated object information on the returns to the brain (including color image), or remarkably participate various dispersion, combination, splicing and other senior specific functions in brain activity. This allows us more generally from superstring point of view to introduce the concept of $p$-brane
[6] structure and try to investigate the possibility of biological brane in the radiation of life.

\section{Experimental Phenomena}

Based on the relevant experimental evidences [7-] from the somatic science past 30 years in china, the main composition of bio-radiation contains special electromagnetic radiation or particle flow which can cause ionization of the film. This sort of radiation can allow film to be sensitive, which is often significantly affected by low-frequency signals (including infrasonic) complex modulation, including amplitude modulation and frequency modulation. The modulation wave itself may be very low frequency of sound, whose amplitude and frequency may change with the environment and the conscious control, and width and shape of beam also have some regular or random changes. When qi is excited it has some characteristics as laser (microwave), such as the focus is very good, and has the very strong direction in the moment, etc. but can be under conscious control or due to changes in certain factors change. In fact, the bio-radiation possesses particle characteristics which are marked with suitable soliton. For example, from the many phenomena of so called RS (somatic science in Chinese meaning) photography $[7,8]$ we can see that the spread mode of motion of the bio-radiation like smoke, fluid, or ultrafine particle flow, which can be vividly controlled by the mind as brush dipped in ink juice, while the ink is as ultrafine particles composed of the bio-radiation, for instance, in RS 
human field photography, only when the film and ultra fine particles of ink direct contact with each others, the film can be sensitive, while if it is too far or too close the film will be not sensitive.

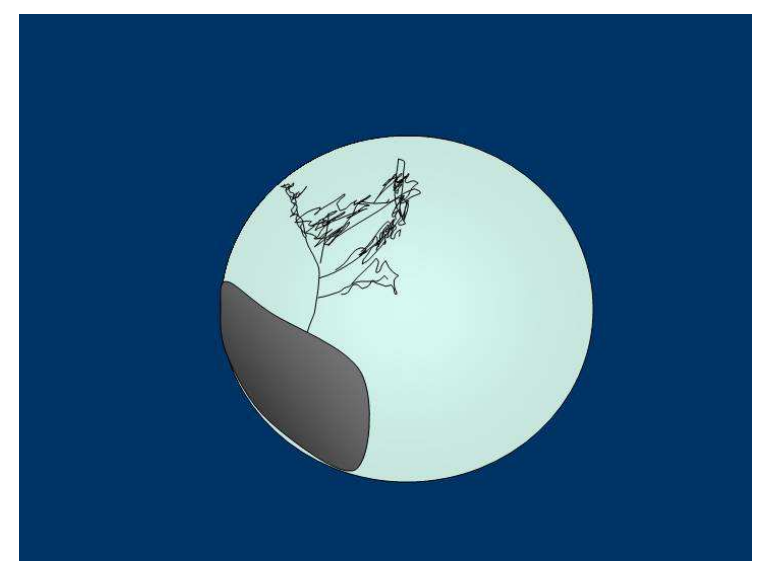

Figure 1. The series of lines can form a tree-like based on a rock-like sub-brane in a 3-brane, which is as a beautiful moon night as shown in RS photography, where the different color area corresponds to the different frequency of the week-electromagnetic radiation. The background gauge field with the week-electromagnetic interaction is demonstrated as blue color, which is not only restricted in the electromagnetic interaction.

\section{3. p-Brane Model}

On the other hand, $p$-brane [9-12] is external object that sweep out a $(p+1)$-dimensional space-time surface called as the world volume $M$, with coordinates $\xi^{m}, m=0,1, \cdots, p$ in a $D$-dimensional background space-time $\underline{M}$ with coordinates $X^{\underline{n}}, \underline{n}=0,1, \cdots, D-1$. Where for the world volume, the coordinates are used $m, n, p, \cdots$, and for the background, the corresponding coordinates are $\underline{m}, \underline{n}, \underline{p}, \cdots$ The surface $M$ swept out by the $p$-brane in the background space-time $\underline{M}$ is described by the function $X^{\underline{n}}\left(\xi^{n}\right)$ which extremes the action as

$$
-T \int d^{p+1} \sqrt{-\operatorname{det} g_{m n}}
$$

where $g_{m n}$ is induced metric on $M$ by

$$
g_{m n}=\partial_{n} X^{\underline{n}} \partial_{m} X^{m} g_{\underline{n} \underline{m}}
$$

$g_{\underline{m} \underline{n}}$ is the metric of the background space-time, and $T$ is the brane tension. This action is invariant under reparameterisations of both $\underline{M}$ and $M$. A $p$-brane naturally couples to an anti-symmetric rank $p+1$ gauge field $A_{\underline{m}_{1} \cdots \underline{m}_{\mathrm{p}+1}}\left(X^{\underline{n}}\right)$ living on the background space-time by the form

$$
q \int d^{p+1} \xi \varepsilon^{n_{1} \cdots n_{p+1}} \partial_{n_{1}} X^{\underline{m}_{1}} \cdots \partial_{n_{p+1}} X^{\underline{m}_{p+1}} A_{\underline{m}_{1} \cdots \underline{m}_{p+1}}\left(X^{\underline{n}}\right)
$$

where $q$ is the coupling constant which is just the charge of the brane.

The background space-time coordinates $\underline{n}, \underline{m}$ can be spitted into those associated with the longitudinal direction of the brane and those related to the transverse direction of the brane. The former is denoted as $n, m, \cdots=0, \cdots, p$, and the latter is expressed as $n^{\prime}, m^{\prime}, \cdots=p+1, \cdots, D-1$. By choosing the static gauge and identifying the $p+1$ longitudinal coordinates $X^{n}(\xi), n=0,1, \cdots, p$ with the coordinates $\xi^{n}, n=0,1, \cdots ; p$ of the $p$-brane world volume as

$$
X^{n}(\xi)=\xi^{n}, \text { for } n=0,1, \cdots, p
$$

which lets the transverse coordinates

$$
X^{n^{\prime}}(\xi)=\xi^{n}, \text { for } n^{\prime}=p+1, \cdots, D-1
$$

describe the dynamics of the brane. Moreover a super- $p$-brane can be viewed as a $(p+1)$-dimensional bosonic sub-manifold $M$, with $M$ coordinates extended as

$$
Z^{\underline{N}}=\left(X^{\underline{n}}, \Theta^{\underline{\alpha}}\right)
$$

where $\Theta^{\underline{\alpha}}$ is a spinor related to fermionic freedom. Then the motion of the super-brane in the background superspace is expressed as $Z^{\underline{N}}\left(\xi^{n}\right)$. There exist super-D-brane, called as Dirichlet (D-) brane, whose dynamics involves a vector field $A_{\mathrm{n}}, n=0,1, \cdots, p$, in the brane world volume and a scaler field for each normal direction of the brane.

Concrete speaking, a D $p$-brane is an extended object with $p$ spatial dimensions. It is a non-perturbative solitonic states of superstring, and is characterized as hypersurface with the Dirichlet boundary condition. D-branes are dynamic objects which is described by the Dirac--Born--Infeld action in the low-energy regime. This sort of nonlinear action is the scalar field corresponding to the transverse position of the D-brane in the background and the U(1) gauge field in its world volume. Generally, the (D-p) states on the D-brane are composites on the D $p$-brane by $(p-1)$ photon states and (D- $p)$ scalar states.

All of these provide the rich and complicated structure to the brane, considering the above evidences of the characteristics of bio-radiation, we introduce a novel concept that the bio-radiation is composed by brane. In fact, from many phenomena of RS photography one can see that the spread mode of motion for bio-radiation like smoke or ultrafine particle flow, here a ultrafine particle can be considered as a 0-brane described in the background space by $Z^{\underline{N}}=\left(\xi^{n}\right)$, for $n=0,1,2,3 ; \underline{N}=4, \cdots, d-1$, where $d$ is given by the concrete model in terms of super-string theory. For example, the dynamics of the D4-Brane, induced by the Dirac-Born-Infeld action is expressed as 


$$
\begin{aligned}
& \left.S=\frac{1}{\left(4 \pi^{2} \alpha^{\prime}\right)^{5} g_{s}} \int d^{10} x \sqrt{-\operatorname{det}\left(\eta_{\mu \nu}+T^{-1} F_{\mu \nu}\right.}\right) \\
& \left.=\frac{T}{g_{s}} \int d^{4} \xi \sqrt{-\operatorname{det}_{0 \leq a, b \leq 3}\left(\eta_{a b}+\partial_{a} x^{m} \partial_{b} x_{m}+2 \pi \alpha^{\prime} F_{a b}\right.}\right)
\end{aligned}
$$

where $T$ is the string tension, and the corresponding equation of motion is

$$
\left(\frac{1}{1-\left(T^{-1} F\right)^{2}}\right)^{v \lambda} \partial_{v} F_{\lambda \mu}=0
$$

which reduces to the Maxwell equation in the field theory limit $\alpha^{\prime} \rightarrow 0$. This sort of D4-Brane can show very rich electro-magnetic structure, such as the D4-Brane only with electric or magnetic fields. Indeed, if a pure electric background is specified by the field strength tensor as

$$
F_{0 i}=E_{i}, F_{i j}=0, \text { for } i, j=0,1,2,3,
$$

then the Born-Infeld in the four dimensional space-time is expressed by

$$
S(E)=\frac{1}{g_{s}}\left(\frac{T}{2 \pi}\right) \int d^{4} x \sqrt{\left(1-\left(T^{-1} E\right)^{2}\right)}
$$

which allows us to see that, at the origin of the source for $\vec{E}$, the electric field arrives at a maximum value[12]

$$
E_{c}=T=\frac{1}{2 \pi \alpha^{\prime}}<|\vec{E}|
$$

While the D-brane only with magnetic field is also possible, here one can find that the magnetic flux is simply the magnetic field multiplied by the area of the fundamental domain of the torus. More generally, this sort of field can be a gauge field with the week-electromagnetic interaction, which is not only restricted in electromagnetic interaction.

Furthermore, if the $N \mathrm{D} p$-branes are parallel such as $N$ parallel D2-branes, then its contained information is quit complicated. In fact, to distinguish the various sectors which consisting of open strings that begin on brane one and end on brane two, the additional ground-state labels integers $[i j]$ is necessary. The first integer expresses the brane on which the $\sigma=0$ endpoint lies, and the second integer demonstrates the brane on which $\sigma=\pi$ endpoint lies, so that the open strings in the $[i j]$ sector extend from brane $i$ to brane $j$, then the ground states are represented as four types:

$$
\left|p^{+}, \vec{p} ;[i i]\right\rangle,\left|p^{+}, \vec{p} ;[j j]\right\rangle,\left|p^{+}, \vec{p} ;[i j]\right\rangle,\left|p^{+}, \vec{p} ;[j i]\right\rangle
$$

Thus the states of open strings in the $[i j]$ sector are constructed from oscillators acting on $\left|p^{+}, \vec{p} ;[i j]\right\rangle$ expressed as

$$
\left[\prod_{n=1}^{\infty} \prod_{i=2}^{p}\left(a_{n}^{i+}\right)^{\lambda_{n, j}}\right]\left[\prod_{m=1}^{\infty} \prod_{\alpha=p+1}^{d}\left(a_{n}^{\alpha+}\right)^{\lambda_{m, \alpha}}\left|p^{+}, \vec{p} ;[i j]\right\rangle\right]
$$

Then the Schrödinger wave function takes the schematic form as

$$
\psi_{i_{1} \cdots i_{p} a_{1} \cdots a_{q}}\left(\tau, p^{+}, \vec{p} ;[i j]\right)
$$

If the density operator is

$$
\rho\left(\tau, p^{+}, \vec{p} ;[i j]\right)=\left|\psi_{i_{1} \cdots i_{p} a_{1} \cdots a_{q}}\right\rangle\left\langle\psi_{i_{1} \cdots i_{p} a_{1} \cdots a_{q}}\right|
$$

then the quantum information density is given by

$$
I\left(\tau, p^{+}, \vec{p} ;[i j]\right)=\rho \ln \rho, \text { for } i, j=0,1, \cdots, N-1
$$

which shows $i, j=0,1, \cdots, N-1$ and provides more complicated informational structure. So the combination of $N$ parallel branes provide a model to support the evidence that the human body radiation can send a quite dense comb waveforms which contain huge capacity of information bits measured by the Sheng Jinchuang [8]. This sort of combination of branes can go further as showing in following sections.

\section{Brane in Brane and Combination}

It is marvelously that the brane can be inside a brane. For example, one can construct a $\mathrm{D}(p$-2)-brane inside a $\mathrm{D} p$-brane as a magnetic vortex or monopole, or a $\mathrm{D}(p-4)$-brane inside a $\mathrm{D} p$-brane as an instanton, and so on [11], where the Chern-Simon action $S_{c s}$ is a fundamental ingredient in the expression of D-brane dynamics which involves the branes in different dimensional space-time as a combination:

$$
\begin{aligned}
& S_{C S}=\frac{N T_{p}}{2} \int_{\Sigma_{p+1}} C^{(p+1)}+\frac{T_{p}}{4 \pi} \int_{\Sigma_{p+1}} C^{(p-1)} \wedge \operatorname{Tr}(F) \\
& +\frac{T_{p}}{8 \pi^{2}} \int_{\Sigma_{p+1}} C^{(p-3)} \wedge \operatorname{Tr}(F \wedge F)+\cdots \\
& +\frac{T_{p}}{2 k !(2 \pi)^{k}} \int_{\Sigma_{p+1}} C^{(p+1-2 k)} \wedge \operatorname{Tr}(\underbrace{F \wedge F \wedge \cdots \wedge}_{k-\text { times }})+\cdots
\end{aligned}
$$

where $C$ is the total Ramond-Ramond potential, and $F$ is the exponential of the curvature two-form. Each term in the above series has a significant meaning: the first term expresses the usual $p$-brane charge with $\operatorname{Tr} 1=N$ appropriate to $N$ coincident $\mathrm{D} p$-branes, the second term coupling with the Ramond-Ramond potential $C^{(p-1)}$ and carries (p-2)-brane charge,

$$
\frac{1}{2 \pi} \int_{S_{\infty}^{2}} \operatorname{Tr}(F)
$$


where $S_{\infty}^{2}$ is a two-dimensional sphere at infinity inside the original $\mathrm{D} p$-brane world volume $\Sigma_{p+1}$, and in a similar way, the third term corresponds to ( $p-4)$-brane charge

$$
\frac{1}{8 \pi^{2}} \int_{S_{\infty}^{4}} \operatorname{Tr}(F \wedge F)
$$

as induced a non-trivial instanton; furthermore the $k$-th term represents $(p-2 k)$-brane charge is

$$
\frac{1}{2 k !(2 \pi)^{k}} \int_{S_{\infty}^{2 k}} \operatorname{Tr}\left(F \wedge^{k} F\right)
$$

This means if the original brane with $p=3$, then the inside sub-brane is $p-0=3$ or $p-2=1$. Their combination may design as the "moon" plus "line" fashions as described in the RS photography taken by the Sheng Jinchuang and Sun Chuling [8], which is simulated in Fig.1. Here the series of 1-brane lines can form a tree-like, which is based on a rock-like 3-sub-brane, and included in a moon like 3-brane. The different color sections correspond to the different frequency of week-electromagnetic radiation or charges. Here the background gauge field with the week-electromagnetic interaction is demonstrated as blue color. So this brane in brane structure forms a beautiful scene as a peaceful moon night. But we want to emphasize that this picture is not to reflect nature but the bio-radiation controlled by the brain! Furthermore, in the extension vein, if 0-brane as point, 1-brane as line, 2-brane as face, and $p$-brane as geometric form, then the combination of all branes in branes can simulate the various of pictures from the RS photography

\section{Conclusion}

In conclusion, the brane in brane structure for bio-radiation has been presented, which makes sense by comparing with the RS photography from the somatic sciences. That means the interaction of bio-radiation is not only electromagnetic but the week and electromagnetic. The dimensions may be higher than four. As extension of the solitons, the brane or combination of branes is proposed to play an important role in the bio-radiation systems, which can be used to explain many complicated phenomena from the experiments of the somatic sciences.

\section{References}

[1] Pang Xiao-Feng, and Feng Yuan-Ping, Quantum Mechanics In Nonlinear Systems, World Scientific Publishing Co. Pte. Ltd. Singapore, 2005.

[2] Bi Qiao, Song Kongzhi and H. E. Ruda, Characteristics of coherence and information for the Davydov soliton field, J. Modern Phys. 3, 1907-1913 2012.

[3] Bi Qiao, Song Kongzhi, Zhang Bao Sheng, Breakthrough space obstacle, American Journal of Modern Physics 2, 227-233 2013.

[4] Bi Qiao, Bio-Solitons Return Life, International Review of Physics 3, 278-288 2009.

[5] Bi Qiao, and Song Kongzhi, Macroscopic Quantum Tunneling, Journal of Modern Physics 4, 49-55 2013.

[6] Peter West, Introduction to Strings and Branes, Cambridge University, New York, 2012.

[7] Shen Jinchuang, RS photograph, Chinese J Somatic Science 1, 131-132 (1991). The author studied the bio-radiation from the Sun Chulin acupoints, he found not only the strength can be measured clearly, but also the spatial distribution of different bio-radiation match different functional state with different geometry shape specificity, so that the corresponding parts for photosensitive film directly shows a clear human biological radiation field photograph, referred to as RS photography, where RS means somatic science in Chinese sense.

[8] Shen Jinchuan, Examination and thinking of soul-controlled energy gathering by Sun Chulin, Chinese J Somatic Science 6 , 10-15 (1996).

[9] Nicholas Manton, and Paul Sutcliffe, Topological Solitons, Cambridge University, Cambridge, 2004.

[10] Luis E. Ibánez, and Angel M. Uranga, String Theory and Particle Physics: an introduction to string phenomenology, Cambridge University, Cambridge, 2012.

[11] Richard J. Szabo, An Introduction to String Theory and D-Brane Dynamics, Imperial College Press, London, 2004.

[12] Barton Zwiebach, A First Course in String Theory, Scond Edit, Cambridge University, Cambridge, 2009. 